\title{
Development of a server for a portable near-infrared spectroscopy laboratory
}

Brais Galdo, Daniel Rivero, Enrique Fernandez-Blanco

Knowing the chemical composition of a substance provides a great deal of information about it. Non-destructive analysis techniques can be used to try to know its chemical composition. Among them, near-infrared spectroscopy (NIRS) stands out. The devices used to obtain this information are known as spectrophotometers.

During the development of the Final Degree Project, an application for cell phones was implemented that connected to one of these devices to take measurements and store them in different databases. In addition, this application also allowed the execution of neural network models based on TensorFlow.

The training of models and the processing of the databases generated by the application is not feasible due to the characteristics of cell phones. This is why we have tried to solve this problem in this Master Thesis by developing a web server with a dedicated architecture based on microservices. This architecture tries to make the most of the characteristics of the different machines that contain it, dividing it into modules that cover only the needs of each one, avoiding the use of resources that are not necessary. The tasks carried out in the system are developed offline, avoiding the need for the user to stay connected while the different processes are executed. It has been necessary to divide the system into 2 large blocks taking into account a division based on functionalities:

The front-end is composed of a web page that enables the realization of all the functionalities in a simple way and is adapted to different devices, such as mobiles, tablets or computers.

The back-end is composed of 3 modules: user authentication and file management, model training and database processing. Both the model training module and the data processing module make use of the configuration files generated in the front-end part of the system. These modules are contained so that, if it is necessary to add computational capacity, it is sufficient to replicate the indicated service without consuming unnecessary resources. 